\title{
The effect of entomopathogenic fungal culture filtrate on the immune response of the greater wax moth, Galleria mellonella
}

\author{
Louise Mc Namara*, James C. Carolan, Christine T. Griffin, David Fitzpatrick, Kevin Kavanagh \\ Department of Biology, Maynooth University, Maynooth, Kildare, Ireland
}

\section{A R T I C L E I N F O}

\section{Keywords:}

Proteomics

Immune response

Antimicrobial peptide

Prophenoloxidase

Biocontrol

Entomopathogenic fungi

\begin{abstract}
A B S T R A C T
Galleria mellonella is a well-established model species regularly employed in the study of the insect immune response at cellular and humoral levels to investigate fungal pathogenesis and biocontrol agents. A cellular and proteomic analysis of the effect of culture filtrate of three entomopathogenic fungi (EPF) species on the immune system of G. mellonella was performed. Treatment with Beauveria caledonica and Metarhizium anisopliae $96 \mathrm{~h}$ culture filtrate facilitated a significantly increased yeast cell density in larvae (3-fold and 3.8-fold, respectively). Larvae co-injected with either M. anisopliae or B. caledonica culture filtrate and Candida albicans showed significantly increased mortality. The same was not seen for larvae injected with Beauveria bassiana filtrate. Together these results suggest that $B$. caledonica and $M$. anisopliae filtrate are modulating the insect immune system allowing a subsequent pathogen to proliferate. B. caledonica and $M$. anisopliae culture filtrates impact upon the larval prophenoloxidase (ProPO) cascade (e.g. ProPO activating factor 3 and proPO activating enzyme 3 were increased in abundance relative to controls), while $B$. bassiana treated larvae displayed higher abundances of alpha-esterase when compared to control larvae (2.4-fold greater) and larvae treated with $M$. anisopliae and B. caledonica. Treatment with EPF culture filtrate had a significant effect on antimicrobial peptide abundances particularly in $M$. anisopliae treated larvae where cecropin-D precursor, hemolin and gloverin were differentially abundant in comparison to controls. Differences in proteomic profiles for different treatments may reflect or even partially explain the differences in their immunomodulatory potential. Screening EPF for their ability to modulate the insect immune response represents a means of assessing EPF for use as biocontrol agents, particularly if the goal is to use them in combination with other control agents. Additionally EPF represent a valuable resource pool in our search for natural products with insect immunomodulatory and biocontrol properties.
\end{abstract}

\section{Introduction}

Beauveria bassiana and Metarhizium anisopliae are amongst the best characterised and widely used entomopathogenic fungi (EPF). These EPF have a global distribution, infect a wide range of insects and are used to control vectors of human disease and plant pests (Shah and Pell, 2003; Glare et al., 2008; Gao et al., 2011; Xiao et al., 2012; Lacey et al., 2015; Butt et al., 2016). EPF have great potential as biopesticides since, unlike bacteria and viruses, they infect hosts through direct cuticle penetration, allowing them to act as contact insecticides (Leger et al., 2011). In addition to their role as insect pathogens, EPF have a crucial role in natural ecosystems as endophytes, plant disease antagonists, rhizosphere colonizers, and plant growth promoters (Gao et al., 2011; Lacey et al., 2015). Despite being promising biocontrol agents EPF have not fulfilled their potential and often fail when tested in the field.
Biocontrol agents are generally slower acting than their chemical counterparts and their high specificity may necessitate the application of more than one agent to control a number of pests. To improve efficacy, biocontrol agents can be combined to increase the success of the application. Combining treatments may result in additive, antagonistic or synergistic interactions. If a combination resulted in a synergistic interaction, then the associated cost of EPF may be reduced. Combinations of EPF, especially $M$. anisopliae, with various species of entomopathogenic nematodes has resulted in synergy in lab and field trials against various insect pests (Ansari et al., 2006, 2008, 2010; Anbesse et al., 2008). Synergism can also occur when different species of EPF are applied together; Metarhizium flavoviride and B. bassiana can be combined to overcome the constraints of temperature in controlling thermoregulating grasshoppers (Inglis et al., 1997). In the case of synergism, combined applications may make the host more susceptible

\footnotetext{
Abbreviations: PO, phenoloxidase; PPO, prophenoloxidase; EPF, entomopathogenic fungi; PCA, principal component analysis; AMP, antimicrobial peptide

* Corresponding author at: Teagasc, Oak Park, Crop Research Centre, Co., Carlow, Ireland.

E-mail address: Louise.McNamara@teagasc.ie (L. Mc Namara).
} 
through modulating their immune system, prolonging developmental stages or by the two treatments acting on different components of the host population (Lacey et al., 2015).

Knowledge of the insect immune response induced by fungal pathogens contributes to the understanding of both insect defenses and the fungal pathogenicity that defeats them. Ultimately uncovering fungal virulence determinants may give rise to opportunities to manipulate these virulence factors to improve the success of biocontrol agents (Gillespie et al., 2000). Despite the importance of EPFs as biocontrol agents and the cost benefits of achieving synergy through combinations of agents our understanding of the molecular basis by which EPF modulate the host immune response making it more susceptible to secondary pathogens is still limited.

Host colonization by EPF requires the ability to cope with host immune defenses and extract nutrients from the host (Gillespie et al., 2000) which is achieved through immune evasion by cryptic forms or immune system modulation through the action of secreted molecules (Schrank and Vainstein, 2010). Metarhizium spp. produce a diverse range of enzymes and secondary metabolites that are active against insects, fungi, bacteria, viruses and cancer cells (Roberts and St Leger, 2004; Gao et al., 2011). Metarhizium produce metabolites that are toxic to a broad range of targets, most notably the cyclic hexadepsipeptidic destruxins (Schrank and Vainstein, 2010) which display antiviral, antitumor, insecticidal, cytotoxic, immunosuppressive, phytotoxic and anti-proliferate effects (Kershaw et al., 1999; Sowjanya Sree et al., 2008; Liu and Tzeng, 2012). Metarhizium isolates that produce higher amounts of destruxin are generally more virulent (Amiri-Besheli et al., 2000; Sowjanya Sree et al., 2008). B. bassiana is known to produce cyclic peptides that are cytotoxic and immunosuppressive (Hung et al., 1993) and a diverse selection of secondary metabolites including nonpeptide pigments and polyketides (e.g. oosporein), non-ribosomally synthesized peptides (e.g. beauvericin) and secreted metabolites that have roles in pathogenesis and virulence (Xiao et al., 2012). These metabolites have insecticidal properties and can also inhibit growth of other microorganisms (van der Weerden et al., 2013). B. bassiana products have both realized and proposed applications in industry, pharmaceuticals and agriculture.

A cellular and proteomic analysis of the effect of culture filtrate of three EPF species on the immune system of the lepidopteran greater wax moth Galleria mellonella was performed. G. mellonella is a wellestablished model species and is regularly employed in the study of the insect immune response at cellular (Vilcinskas et al., 1997a, 1997b; Vilcinskas and Götz, 1999) and humoral (Vilcinskas and Matha, 1997; Bergin et al., 2006; Wojda and Jakubowicz, 2007; Wojda et al., 2009) levels. Of greater relevance is the use of $G$. mellonella as a model to investigate fungal pathogenesis and biocontrol agents (Vilcinskas et al., 1997a; Cotter et al., 2000, Fröbius et al., 2000, Bergin et al., 2003; Scully and Bidochka, 2005) and although different insect species may vary greatly in their susceptibility to fungal toxins (Gillespie et al., 2000), Galleria is an excellent substitute for insects that are difficult to source or cultivate, particularly for preliminary investigations. The aim of this work was to investigate the immunomodulatory potential of EPF in order to assess their potential use and mode of action as synergists in combined applications with other control agents.

The three species of EPF chosen were M. anisopliae, B. bassiana and $B$. caledonica. Beauveria caledonica was found to be a naturally occurring pathogen of the pine bark beetles Hylastes ater and Hylurgus ligniperda in New Zealand (Glare et al., 2008; Reay et al., 2008) and the large pine weevil, Hylobius abietis in Ireland (Glare et al., 2008; Williams et al., 2013). Considering B. caledonica kills these pests in their natural environment, it makes this fungus an excellent candidate as a biocontrol agent of coleopteran forestry pests (Glare et al., 2008; Williams et al., 2013).

\section{Materials and methods}

\subsection{Fungal origin and entomopathogenic fungal culture filtrate production}

A commercial strain of $M$. anisopliae (Met52) produced by Novozymes (Denmark) was used. Met 52 was purchased on rice grains from National Agrochemical Distributors, Lusk, Dublin. B. bassiana experimental strain 1694, was supplied by Becker Underwood (Littlehampton, UK). B. caledonica (2c7b) is a native strain isolated from a soil sample from soil close to a pine stump in a felled forest in Hortland, Co. Kildare (Ireland). The soil sample was baited with $G$. mellonella larvae and fungus from the infected cadaver was identified through DNA sequencing of an ITS PCR product (a region of the internal transcribed spacer unit of the ribosomal DNA, IST4, was amplified by PCR). EPF were cultured in Sabouraud Dextrose liquid medium (Oxoid) for $48 \mathrm{~h}, 72 \mathrm{~h}$ and $96 \mathrm{~h}$ in a shaking incubator at $25^{\circ} \mathrm{C}$ and $250 \mathrm{rpm}$. After each time point the culture was filtered through $0.45 \mu \mathrm{m}$ syringe filters and then through $0.2 \mu \mathrm{m}$ syringe filters (Sartstedt). The filtrate was collected and stored at $-80^{\circ} \mathrm{C}$.

\subsection{Inoculation of Galleria mellonella larvae}

Sixth instar larvae of G. mellonella (Mealworm Company, Sheffield, England) were stored in the dark at $15{ }^{\circ} \mathrm{C}$ in wood shavings prior to use. Larvae were stored immediately upon receipt from the supplier. Larvae that had been stored for 1-3 weeks and weighing 0.24-0.28 g were used. Larvae were inoculated with $20 \mu$ of culture filtrate through the last pro-leg using a Myjector U100 insulin syringe (Terumo Europe, Leuven, Belgium). Control larvae were inoculated with $20 \mu \mathrm{l}$ of Sabouraud dextrose liquid medium. Larvae were incubated at $20^{\circ} \mathrm{C}$.

\subsection{Enumeration of haemocyte and yeast cell densities, and infection susceptibility assays}

The density of circulating haemocytes in larvae was assessed as described previously (Bergin et al., 2003). All experiments were performed on three independent occasions.

To test the effect of EPF on the immune response to a subsequent infection, larvae were inoculated with EPF culture filtrate or Sabouraud dextrose (control) and incubated for $24 \mathrm{~h}$ at $20^{\circ} \mathrm{C}$, after which they received a second inoculation through the second proleg with Candida albicans $\left(10^{5}\right.$ cells in $\left.20 \mu \mathrm{l}\right)$. Candida albicans MEN (serotype B, wildtype originally isolated from an eye infection (a gift from Dr. D. Kerridge, Cambridge, UK) was cultured to the stationary phase overnight in yeast extract peptone dextrose (YEPD) at $30{ }^{\circ} \mathrm{C}$ and $200 \mathrm{rpm}$ on an orbital shaker (Browne et al., 2015). Following the second inoculation, larvae were incubated for a further $24 \mathrm{~h}$ or $48 \mathrm{~h}$ at $20^{\circ} \mathrm{C}$ and were homogenized in $3 \mathrm{ml}$ of sterile PBS. After serial dilution in PBS, $100 \mu \mathrm{l}$ of each sample was spread on YEPD plates containing erythromycin $(1 \mathrm{mg} / \mathrm{ml})$. The plates were incubated for $48 \mathrm{~h}$ at $30^{\circ} \mathrm{C}$. Yeast cell density was subsequently calculated per larva.

To test whether EPF would make larvae more susceptible to a second pathogen, larvae were inoculated through the last proleg with $20 \mu \mathrm{l}$ of culture filtrate or Sabouraud dextrose and incubated at $20^{\circ} \mathrm{C}$. After $24 \mathrm{~h}$ larvae were given a second injection with $C$. albicans $\left(1 \times 10^{5} / 20 \mu\right.$ l, culture as above), or PBS. There were ten larvae per treatment. Larvae were incubated at $20{ }^{\circ} \mathrm{C}$ and mortality was recorded for up to 14 days.

\subsection{Protein sample preparation and mass spectrometry}

Larvae were injected with $20 \mu \mathrm{l}$ of culture filtrate or Sabouraud dextrose (controls) and incubated for $48 \mathrm{~h}$ at $20^{\circ} \mathrm{C}$. Five larvae per treatment were bled into a pre-chilled $1.5 \mathrm{ml}$ centrifuge tube and spun at $1500 \mathrm{~g}$ for $5 \mathrm{~min}$ at $4{ }^{\circ} \mathrm{C}$. Samples were diluted in PBS and a Bradford assay was carried out. Protein $(100 \mu g)$ was removed to a pre-chilled 
A

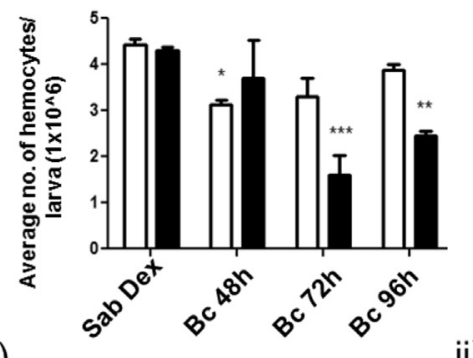

i)

ii)

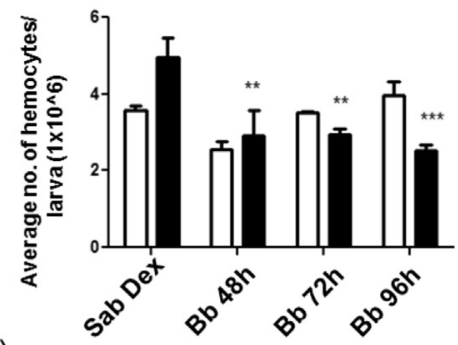

B

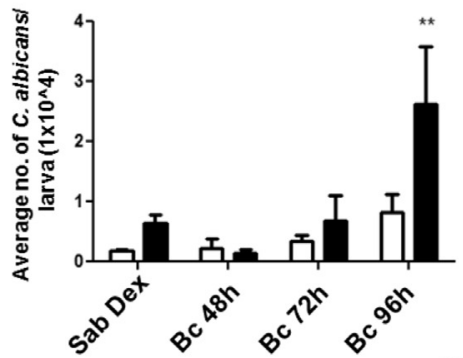

i)

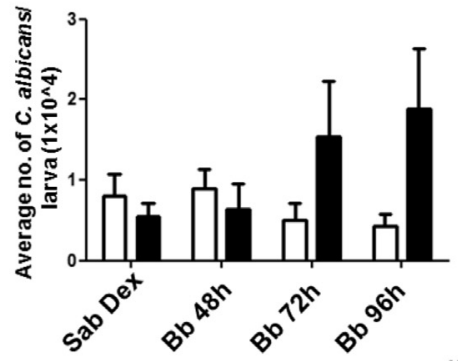

ii)

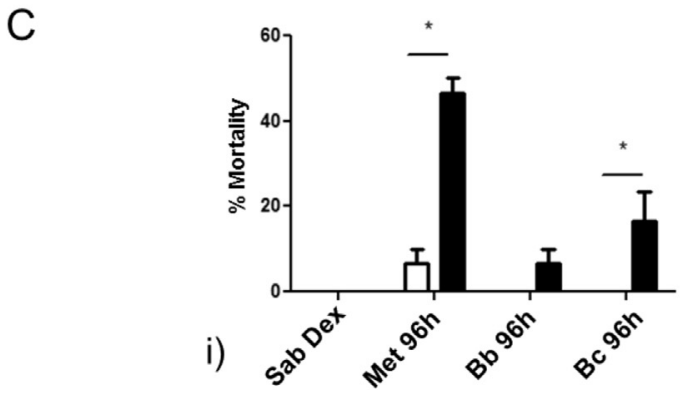

ii)

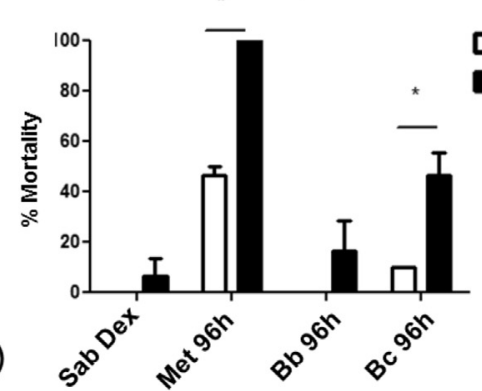

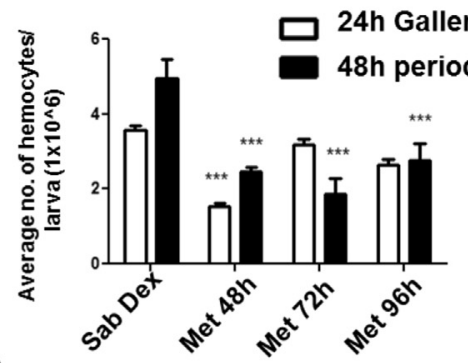

iii)

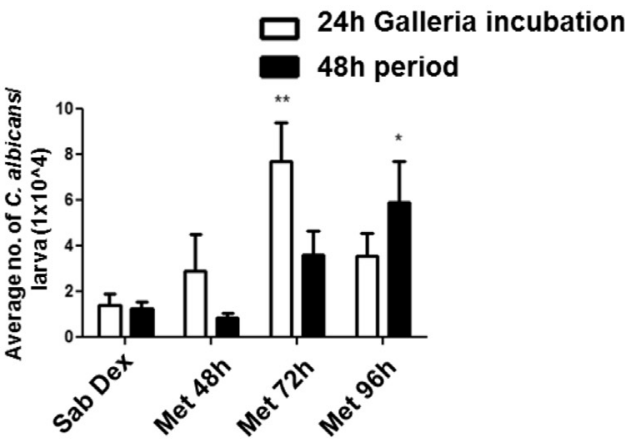

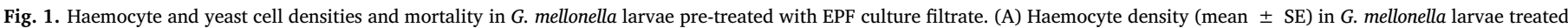

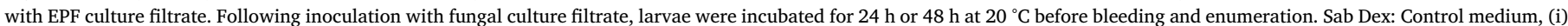

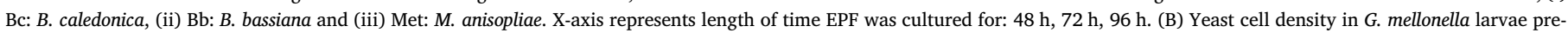

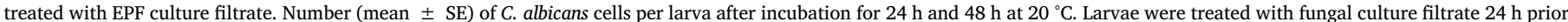

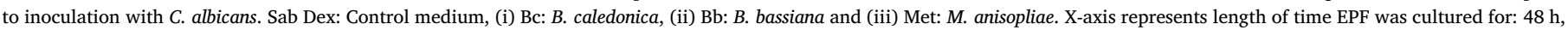

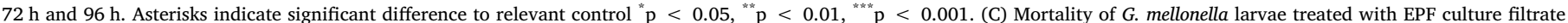

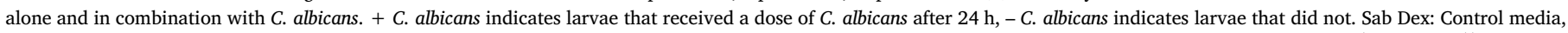

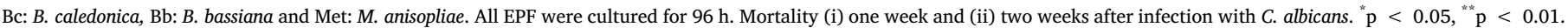

$1.5 \mathrm{ml}$ centrifuge tube and ice cold $100 \%$ acetone was added at ratio of 1:3 (sample:acetone). Protein was precipitated at $-20^{\circ} \mathrm{C}$. The sample was centrifuged at $13,000 \mathrm{~g}$ for $10 \mathrm{~min}$ and the protein pellet was resuspended in $100 \mu \mathrm{l}$ of resuspension buffer (6 M urea, $2 \mathrm{M}$ thiourea, $5 \mathrm{mM}$ calcium chloride). Protein $(75 \mu \mathrm{g})$ was reduced with dithiothreitol $(200 \mathrm{mM})$ and alkylated with iodoacetamide $(1 \mathrm{M})$. Samples were digested with sequence grade trypsin (Promega, Ireland) at a trypsin:protein ratio of $1: 40$, overnight at $37^{\circ} \mathrm{C}$. Four replicate samples were prepared for each treatment.

Tryptic peptides were purified for mass spectrometry using C18 spin filters (Medical Supply Company, Ireland) and $1 \mu \mathrm{g}$ of peptide mix was eluted onto a Q Exactive (ThermoFisher Scientific, U.S.A) high resolution mass spectrometer connected to a Dionex Ultimate 3000 (RSLCnano) chromatography system. Peptides were separated by an increasing acetonitrile gradient (2-40\%) on a Biobasic C18 PicofritTM column ( $100 \mathrm{~mm}$ length, $75 \mathrm{~mm}$ ID), using a $120 \mathrm{~min}$ reverse phase gradient at a flow rate of $250 \mathrm{~nL} / \mathrm{min}$. All data were acquired with the mass spectrometer operating in automatic data dependent switching mode. A full MS scan at 140,000 resolution and a scan range of $300-1700 \mathrm{~m} / \mathrm{z}$ was followed by an MS/MS scan, at resolution 17,500 , to select the 15 most intense ions prior to MS/MS.

\subsection{Quantitative mass spectrometry data analysis}

Protein identification from the MS/MS data was performed using the Andromeda search engine in MaxQuant (version 1.2.2.5; http:// maxquant.org/) to correlate the data against a 6-frame translation of the EST contigs for G. mellonella (Vogel et al., 2011). The following search parameters were used: first search peptide tolerance of $20 \mathrm{ppm}$, second search peptide tolerance $4.5 \mathrm{ppm}$ with cysteine carbamidomethylation as a fixed modification and $\mathrm{N}$-acetylation of protein and oxidation of methionine as variable modifications and a maximum of 2 missed cleavage sites allowed. The MS proteomics data and MaxQuant search output files have been deposited to the ProteomeXchange Consortium (Côté et al., 2012) via the PRIDE partner repository with the dataset identifier PXD005939. Results processing, statistical analyses and graphics generation were conducted using Perseus v. 1.5.0.31. Label free quantification (LFQ) intensities were $\log _{2}$-transformed and $t$ tests comparing EPF treated larvae with controls were performed using a p-value of 0.05 . Proteins were kept in the analysis if they were found in all 4 replicates in at least one group. Principal component analysis (PCA) was used to emphasize variation and visualize strong patterns in the data. Proteins found to be absent (below the level of detection) in one or more treatments and present (above the level of detection) in 
three or fewer treatments were termed 'uniquely detected proteins'. These proteins were also used in statistical analysis of the total differentially expressed group following imputation of the zero values with values that simulate low abundant proteins. These values were chosen randomly from a distribution specified by a downshift of 2.19 times the mean standard deviation (SD) of all measured values and a width of 0.36 times this SD. To obtain an overall proteomic profile of abundance for all significantly expressed and exclusive proteins, hierarchical clustering on Z-score normalised intensity values was performed and the relative protein expression values were displayed as a heat map. The Blast2Go suite (www.blast2go.com) of software tools was utilized to assign gene ontology terms (GO terms) relating to biological processes, molecular function and cellular components.

\subsection{Statistical analysis}

Statistical analysis was carried out using Minitab V.16 statistical software and GraphPad Prism V.5. All data were first tested for normality, where data were found not to be normal, the data were transformed before further analysis was carried out. For alterations to haemocyte densities and yeast loads, data were analysed separately for each EPF species using two-way ANOVA with EPF culture time and assessment time ( 24 or $48 \mathrm{~h}$ post injection) as the factors. Bonferroni post hoc tests were used to compare EPF treatments to relevant controls. To determine whether EPF culture filtrate increases susceptibility of $G$. mellonella to a subsequent infection, data for yeast-injected and PBS-injected larvae were compared using paired $T$-tests.

\section{Results}

\subsection{Alterations in haemocyte densities following injection of larvae with EPF culture filtrate}

Larvae injected with all three EPF culture filtrates showed a significant alteration in haemocyte densities at $24 \mathrm{~h}$ and $48 \mathrm{~h}$ (Fig. 1A). Administration of all three EPF induced a decline in the haemocyte densities of larvae following inoculation with $72 \mathrm{~h}$ or $96 \mathrm{~h}$ culture filtrate and incubation for $48 \mathrm{~h}$, relative to their appropriate controls. There was also a significant interaction between treatment and time in all EPF tested; treatments perform differently after different incubation periods.

\subsection{Alterations in yeast cell density following injection of larvae with EPF culture filtrate}

In larvae injected with $B$. caledonica culture filtrate, treatment had a significant effect on yeast cell density $\left(\mathrm{F}_{3,16}=5.01, \mathrm{p}<0.05\right)$. Inoculation with $B$. caledonica $96 \mathrm{~h}$ culture filtrate resulted in a significant difference $(\mathrm{p}<0.01)$ in yeast cell density, with a 3 -fold increase relative to controls (Fig. 1B i), when the larvae were incubated for $48 \mathrm{~h}$ following injection with $C$. albicans. In larvae injected with $M$. anisopliae culture filtrate, treatment had a significant effect on yeast load $\left(\mathrm{F}_{3,16}=0.16, \mathrm{p}<0.001\right)$. Treatment with $M$. anisopliae $96 \mathrm{~h}$ culture filtrate induced a significant alteration in yeast load $(\mathrm{p}<0.05)$ with a fold increase of 3.76 relative to controls, when the larvae were incubated for $48 \mathrm{~h}$ following injection with C. albicans (Fig. 1B iii). Additionally, treatment with $M$. anisopliae $72 \mathrm{~h}$ culture filtrate caused a 4.42-fold increase ( $\mathrm{p}<0.01$ ) when the larvae were incubated for $24 \mathrm{~h}$ following injection with $C$. albicans. In contrast, treatment with $B$. bassiana ( $48 \mathrm{~h}, 72 \mathrm{~h}, 96 \mathrm{~h}$ culture filtrate) did not cause a significant alteration in yeast cell density in larvae relative to controls (Fig. 1B ii).

\subsection{Effect of EPF culture filtrate on susceptibility of larvae to subsequent infection}

More than $40 \%$ of larvae died within two weeks of injection with $M$. anisopliae culture filtrate alone, while all other single species infections (B. bassiana, B. caledonica or C. albicans only) resulted in negligible mortality (Fig. 1C ii). Larvae that were treated with $M$. anisopliae or $B$. caledonica culture filtrate before treatment with $C$. albicans had higher mortality than larvae treated with either culture filtrate or $C$. albicans alone. The difference between treatments with and without $C$. albicans was significant one week $(M$. anisopliae: $\mathrm{T}=-6.93, \mathrm{p}<0.05$; $\mathrm{B}$. caledonica: $\mathrm{T}=-4.74, \mathrm{p}<0.05$; Fig. $1 \mathrm{C}$ i) and two weeks after $C$. albicans infection $(M$. anisopliae: $\mathrm{T}=7, \mathrm{p}<0.05 ; B$. caledonica: $\mathrm{T}=-4.75, \mathrm{p}<0.05$; Fig. 1C ii) with $M$. anisopliae and C. albicans combined infection causing $100 \%$ mortality after two weeks. This pattern was not seen with $B$. bassiana.

\subsection{LFQ analysis of $G$. mellonella larval hemolymph following EPF culture filtrate treatment}

Haemolymph of $G$. mellonella injected with EPF culture filtrate was subjected to LFQ proteomics to assess differences in protein abundance between treated and control larvae. In total, 100 proteins were identified with two or more peptides. Sixty-one of these proteins were either significantly altered in abundance or uniquely detected across the four treatments analysed (Supp. Table $1 \mathrm{a}$ and $\mathrm{b}$ ). Ten proteins were found to be absent (below the level of detection) in one or more treatments and present (above the level of detection) in three or less sample groups. These proteins were termed 'uniquely detected proteins' (Table 1). These proteins were also used in statistical analysis of the total differentially expressed group following imputation (Fig. 3, Table 2) which resulted in the replacement of absent values (represented by NaNs or 0 ) with values that simulated low expression values. This permitted the treatment and visualisation of exclusively expressed and statistically significant proteins simultaneously. PCA was used to visualize the variation in protein abundances across all individual

Table 1

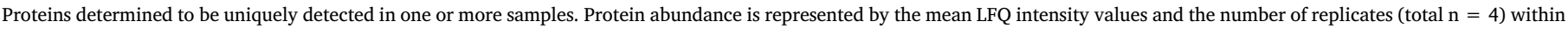
which each protein was detected are given. n.d. = not detected.

\begin{tabular}{|c|c|c|c|c|c|c|c|c|}
\hline \multirow[t]{2}{*}{ Protein Annotation } & \multicolumn{4}{|c|}{ Mean LFQ Intensities ( $\log _{2}$ transformed) } & \multicolumn{4}{|c|}{ Number of replicates } \\
\hline & Control & BB & $\mathrm{BC}$ & MET & Control & BB & $\mathrm{BC}$ & MET \\
\hline Apyrase & $4.5 \times 10^{7}$ & $8.3 \times 10^{7}$ & 0 & $7.3 \times 10^{7}$ & 2 & 3 & 0 & 4 \\
\hline Cecropin-D precursor & $1.4 \times 10^{8}$ & $1.6 \times 10^{8}$ & $3.3 \times 10^{8}$ & 0 & 3 & 3 & 4 & 0 \\
\hline Peptidoglycan recognition-like & $8.8 \times 10^{7}$ & $8.1 \times 10^{7}$ & $2.5 \times 10^{8}$ & 0 & 3 & 4 & 4 & 0 \\
\hline Aminoacylase-1-like & $1.9 \times 10^{8}$ & 0 & $4.8 \times 10^{7}$ & 0 & 4 & 0 & 1 & 0 \\
\hline Prophenoloxidase activating factor 3 & 0 & $6.7 \times 10^{7}$ & $4.6 \times 10^{8}$ & $6.8 \times 10^{8}$ & 0 & 2 & 4 & 4 \\
\hline Gustatory receptor candidate 25 & 0 & $4.9 \times 10^{8}$ & $3.3 \times 10^{8}$ & $7.9 \times 10^{8}$ & 0 & 4 & 3 & 3 \\
\hline Contig22050_1 & 0 & $1.1 \times 10^{7}$ & $1 \times 10^{8}$ & $7.4 \times 10^{7}$ & 0 & 1 & 3 & 4 \\
\hline Beta actin & 0 & $7.8 \times 10^{7}$ & $8.5 \times 10^{7}$ & $5.9 \times 10^{8}$ & 0 & 1 & 1 & 4 \\
\hline Peptidylprolyl isomerase B precursor & 0 & 0 & $3.2 \times 10^{7}$ & $6.5 \times 10^{7}$ & 0 & 0 & 2 & 4 \\
\hline Contig20011_1.r1. & $6.3 \times 10^{7}$ & 0 & 0 & 0 & 4 & 0 & 0 & 0 \\
\hline
\end{tabular}


Table 2

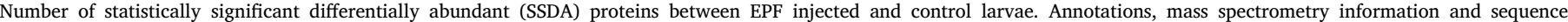

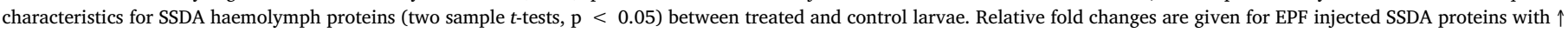
and $\downarrow$ representing higher or lower abundance relative to the controls.

\begin{tabular}{|c|c|c|c|c|c|c|c|c|}
\hline \multirow[t]{2}{*}{ Protein Annotation } & \multicolumn{3}{|c|}{ Relative fold change } & \multirow[t]{2}{*}{ Intensity } & \multirow[t]{2}{*}{ MS/MS } & \multirow[t]{2}{*}{ Peptides } & \multirow{2}{*}{$\begin{array}{l}\text { Mol. Weight } \\
\text { (kDa) }\end{array}$} & \multirow[t]{2}{*}{ Sequence length } \\
\hline & BB & BC & MET & & & & & \\
\hline Gustatory receptor candidate 25 & $20.99 \uparrow$ & $10.51 \uparrow$ & $20.97 \uparrow$ & $5.4 \times 10^{9}$ & 46 & 2 & 25.9 & 235 \\
\hline Scolexin & $1.96 \uparrow$ & $2.18 \uparrow$ & $3.21 \uparrow$ & $2.2 \times 10^{10}$ & 224 & 11 & 58.3 & 528 \\
\hline $27 \mathrm{kDa}$ hemolymph protein & $1.68 \uparrow$ & $1.98 \uparrow$ & $3.06 \uparrow$ & $8.1 \times 10^{9}$ & 125 & 7 & 44.7 & 405 \\
\hline Cationic peptide CP8 precursor & $1.84 \downarrow$ & $2.42 \downarrow$ & $2 \downarrow$ & $1.3 \times 10^{11}$ & 246 & 4 & 17.7 & 168 \\
\hline Contig04490_1 & $2.69 \downarrow$ & $1.82 \downarrow$ & $2.92 \downarrow$ & $2.9 \times 10^{9}$ & 119 & 3 & 8.1 & 77 \\
\hline Heat shock protein 25.4 precursor & $2.82 \uparrow$ & - & $3.88 \uparrow$ & $3.1 \times 10^{10}$ & 196 & 11 & 28.4 & 252 \\
\hline Contig_190.0 & $2.21 \uparrow$ & - & $1.54 \uparrow$ & $2.0 \times 10^{10}$ & 173 & 8 & 36.6 & 326 \\
\hline Bombyrin & $1.72 \downarrow$ & - & $1.84 \downarrow$ & $3.3 \times 10^{10}$ & 166 & 7 & 37.3 & 322 \\
\hline Contig20220_1 & $1.97 \downarrow$ & - & $3.5 \downarrow$ & $2.8 \times 10^{10}$ & 162 & 10 & 14.8 & 133 \\
\hline Putative protease inhibitor 4 & $2.66 \downarrow$ & - & $3.9 \downarrow$ & $4.3 \times 10^{10}$ & 172 & 6 & 30.0 & 272 \\
\hline Aminoacylase & $3.8 \downarrow$ & - & $6.1 \downarrow$ & $1.3 \times 10^{9}$ & 30 & 4 & 432 & 46.7 \\
\hline Contig19736_1 & $1.95 \downarrow$ & - & $7.32 \downarrow$ & $2.8 \times 10^{10}$ & 174 & 3 & 15.4 & 161 \\
\hline Serine proteinase & $4.49 \uparrow$ & $3.32 \uparrow$ & - & $1.93 \times 10^{9}$ & 57 & 7 & 52.4 & 477 \\
\hline Prophenoloxidase activating factor 3 & - & $14.9 \uparrow$ & $25.2 \uparrow$ & $2.7 \times 10^{9}$ & 68 & 5 & 25.8 & 233 \\
\hline Contig_962.0 & - & $9.3 \uparrow$ & $8.6 \uparrow$ & $5.2 \times 10^{9}$ & 88 & 12 & 53.6 & 489 \\
\hline Contig_1159.0 & - & $4.5 \uparrow$ & $7.3 \uparrow$ & $1.1 \times 10^{9}$ & 41 & 2 & 35.3 & 330 \\
\hline Protease inhibitor 1 precursor & - & $2.75 \uparrow$ & $6.11 \uparrow$ & $1.42 \times 10^{10}$ & 138 & 4 & 24.3 & 222 \\
\hline Prophenoloxidase activating enzyme 3 & - & $3.47 \uparrow$ & $4.52 \uparrow$ & $5.5 \times 10^{9}$ & 202 & 17 & 72.1 & 649 \\
\hline Peptidoglycan recognition protein $\mathrm{S} 2$ precursor & - & $2.76 \uparrow$ & $2.98 \downarrow$ & $1.46 \times 10^{9}$ & 49 & 2 & 17.8 & 156 \\
\hline Gloverin-like protein & - & $1.83 \uparrow$ & $2.44 \downarrow$ & $9.34 \times 10^{8}$ & 57 & 2 & 20.5 & 189 \\
\hline Alpha-esterase 45 & - & $2.06 \downarrow$ & $2.03 \downarrow$ & $3.77 \times 10^{10}$ & 338 & 13 & 33.6 & 291 \\
\hline Methionine-rich storage protein & - & $2.25 \downarrow$ & $2.39 \downarrow$ & $6.47 \times 10^{11}$ & 823 & 20 & 15.2 & 129 \\
\hline Apolipophorin-2 & - & $1.76 \downarrow$ & $2.88 \downarrow$ & $1.25 \times 10^{11}$ & 288 & 8 & 8.7 & 78 \\
\hline Apolipoprotein D like & $2.46 \uparrow$ & - & - & $2.8 \times 10^{10}$ & 208 & 9 & 41.3 & 372 \\
\hline Alpha-esterase 45 & $2.35 \uparrow$ & - & - & $6.6 \times 10^{9}$ & 104 & 9 & 71.2 & 670 \\
\hline Adhesion related protein, transmembrane & $2.29 \uparrow$ & - & - & $2.2 \times 10^{9}$ & 61 & 3 & 40.4 & 360 \\
\hline Integument esterase 2 precursor & $1.97 \uparrow$ & - & - & $5.6 \times 10^{10}$ & 425 & 11 & 56.5 & 503 \\
\hline Aldo-keto reductase AKR2E4-like & $1.72 \uparrow$ & - & - & $2.6 \times 10^{9}$ & 63 & 2 & 9.3 & 80 \\
\hline Chain A, Structure Of Active Serpin K & $1.62 \uparrow$ & - & - & $1.6 \times 10^{11}$ & 754 & 21 & 50.6 & 451 \\
\hline Contig_5682.0 & $1.75 \downarrow$ & - & - & $1.3 \times 10^{11}$ & 333 & 11 & 28.0 & 245 \\
\hline Contig20011_1 & $2.04 \downarrow$ & - & - & $4.4 \times 10^{8}$ & 24 & 2 & 57.6 & 539 \\
\hline Contig20268_1 & $2.21 \downarrow$ & - & - & $5.7 \times 10^{9}$ & 61 & 9 & 25.2 & 222 \\
\hline Protease inhibitor-like protein & $3.06 \downarrow$ & - & - & $4.2 \times 10^{9}$ & 122 & 2 & 12.7 & 120 \\
\hline Glyceraldehyde-3-phosphate dehydrogenase & $3.9 \downarrow$ & - & - & $3.2 \times 10^{9}$ & 58 & 6 & 47.0 & 441 \\
\hline Serpin $3 a$ & - & $2.88 \uparrow$ & - & $2.73 \times 10^{9}$ & 68 & 8 & 110.4 & 989 \\
\hline Beta-1,3-glucan-binding protein & - & $2.4 \uparrow$ & - & $1.66 \times 10^{9}$ & 44 & 2 & 23.4 & 214 \\
\hline Peptidoglycan recognition-like protein B & - & $2.12 \uparrow$ & - & $9.18 \times 10^{9}$ & 166 & 12 & 47.6 & 430 \\
\hline Insecticyanin & - & $1.65 \downarrow$ & - & $5.42 \times 10^{10}$ & 354 & 14 & 29.0 & 253 \\
\hline Beta Actin & - & - & $14.8 \uparrow$ & $1.9 \times 10^{9}$ & 39 & 6 & 30.1 & 279 \\
\hline Putative defense protein Hdd11 & - & - & $5.55 \uparrow$ & $4.93 \times 10^{9}$ & 114 & 7 & 20.7 & 196 \\
\hline $25 \mathrm{kDa}$ silk glycoprotein & - & - & $4.99 \uparrow$ & $9.25 \times 10^{9}$ & 135 & 9 & 84.6 & 746 \\
\hline Contig22050_1 & - & - & $2.7 \uparrow$ & $5.9 \times 10^{8}$ & 33 & 6 & 48.5 & 422 \\
\hline $27 \mathrm{kDa}$ hemolymph protein & - & - & $2.31 \uparrow$ & $6.88 \times 10^{11}$ & 1181 & 10 & 17.3 & 161 \\
\hline Peptidylprolyl isomerase B precursor & - & - & $1.85 \uparrow$ & $3.1 \times 10^{8}$ & 25 & 3 & 39.1 & 354 \\
\hline Twelve cysteine protein 1 & - & - & $1.63 \uparrow$ & $1.8 \times 10^{10}$ & 185 & 7 & 35.6 & 320 \\
\hline Hemolin & - & - & $1.63 \uparrow$ & $3.96 \times 10^{10}$ & 161 & 6 & 15.3 & 140 \\
\hline Gelsolin & - & - & $1.57 \uparrow$ & $9.89 \times 10^{10}$ & 657 & 21 & 50.7 & 469 \\
\hline Methionine-rich storage protein 2 & - & - & $1.54 \uparrow$ & $2.06 \times 10^{12}$ & 4415 & 68 & 56.7 & 489 \\
\hline Transferrin & - & - & $1.57 \downarrow$ & $3.81 \times 10^{11}$ & 1632 & 58 & 106.3 & 965 \\
\hline Hexamerin & - & - & $1.68 \downarrow$ & $2.5 \times 10^{11}$ & 1194 & 53 & 90.6 & 778 \\
\hline Aarylphorin & - & - & $1.77 \downarrow$ & $1.66 \times 10^{11}$ & 328 & 19 & 19.6 & 168 \\
\hline Sensory appendage protein 1 & - & - & $1.87 \downarrow$ & $9.75 \times 10^{9}$ & 111 & 9 & 22.8 & 203 \\
\hline Imaginal disc growth factor-like protein & - & - & $2.12 \downarrow$ & $1.52 \times 10^{11}$ & 523 & 14 & 23.3 & 208 \\
\hline Contig19502_1 & - & - & $2.14 \downarrow$ & $3.07 \times 10^{10}$ & 603 & 34 & 83.6 & 733 \\
\hline $32 \mathrm{kDa}$ ferritin subunit & - & - & $2.2 \downarrow$ & $3 \times 10^{10}$ & 204 & 9 & 43.0 & 381 \\
\hline Multicystatin and procathepsin F precursor & - & - & $2.21 \downarrow$ & $2.96 \times 10^{10}$ & 164 & 8 & 13.2 & 120 \\
\hline Nimrod B precursor & - & - & $2.75 \downarrow$ & $1.9 \times 10^{10}$ & 146 & 3 & 19.5 & 180 \\
\hline Contig22104 & - & - & $2.96 \downarrow$ & $2.12 \times 10^{9}$ & 42 & 2 & 25.5 & 234 \\
\hline Carboxylesterase & - & - & $4.71 \downarrow$ & $5.83 \times 10^{9}$ & 52 & 6 & 30.2 & 272 \\
\hline
\end{tabular}

replicates (Fig. 2A). Four distinct clusters were resolved representing each treatment, with each replicate resolving into their appropriate treatment. $M$. anisopliae was more divergent than the other EPF treatments relative to control larvae. Hierarchical clustering resolved four distinct clusters of proteins with similar expression profiles (Fig. 2B). Cluster A comprises proteins with higher levels of abundance in larvae treated with $M$. anisopliae and $B$. caledonica relative to control larvae and intermediate intensities in B. bassiana treated larvae. This cluster includes a number of proteins involved in the prophenoloxidase (ProPO) cascade; ProPO activating factor and enzyme 3. Cluster B comprises proteins with lower levels of abundance in larvae treated with $B$. bassiana relative to all other treatments and control larvae. Cluster C comprises proteins with lower levels of abundance in larvae treated with $M$. anisopliae relative to all other treatments and control 
A

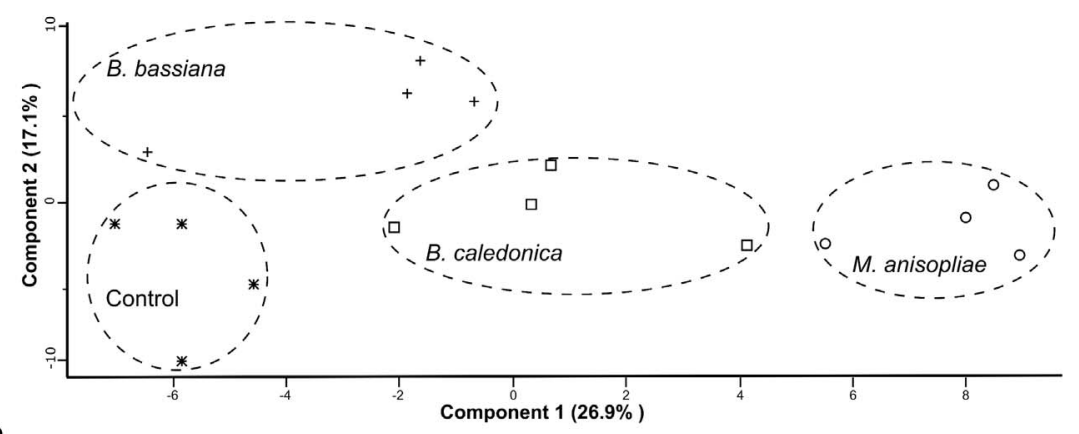

B

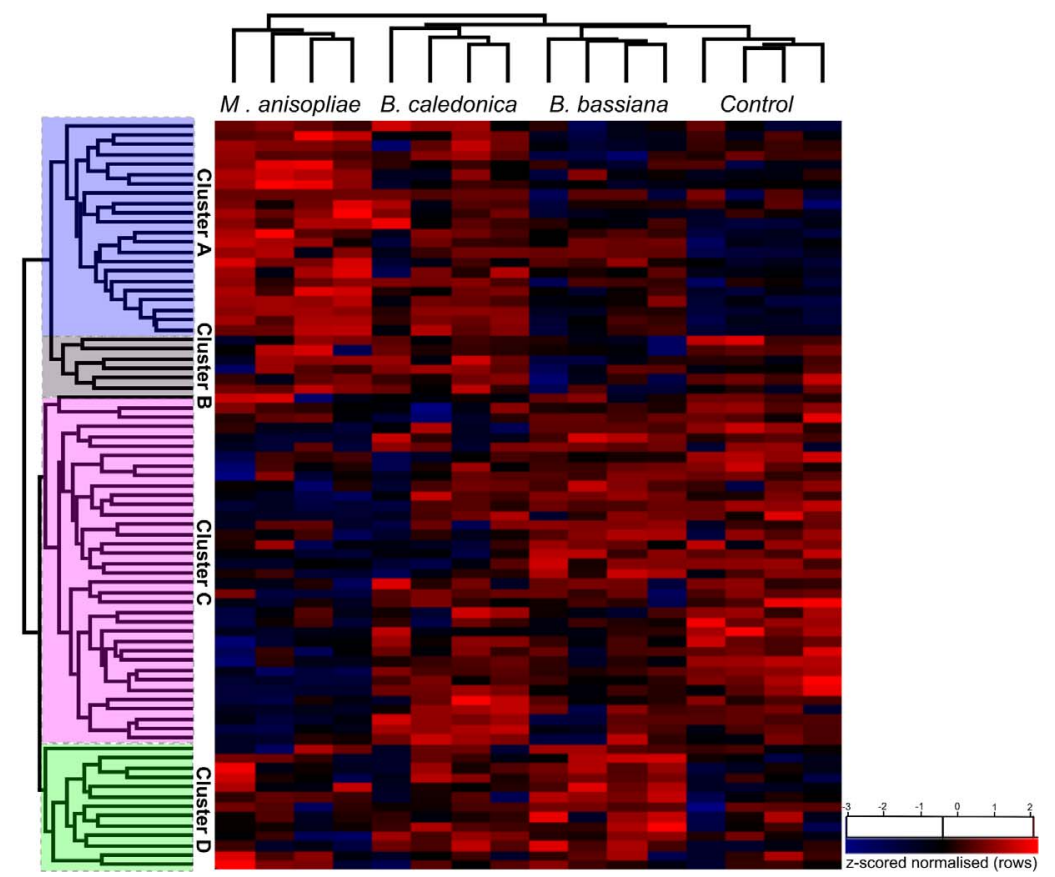

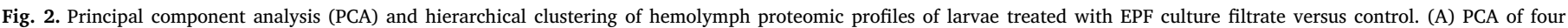

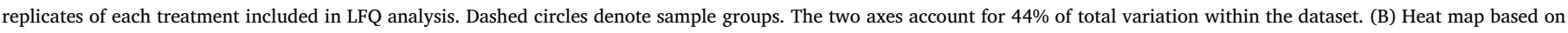

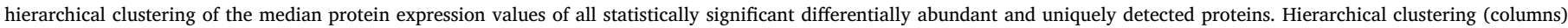
resolved four distinct clusters comprising the replicates from their original sample groups and four protein clusters (rows) based on expression profile similarities.

larvae, including proteins involved in recognition; and peptidoglycan recognition-like protein. Cluster D comprises proteins with higher levels of abundance in larvae treated with $B$. bassiana relative to all other treatments and control larvae.

Blast2GO annotation software (www.blast2GO.com) was used to group proteins based on conserved gene ontology (GO) terms in order to identify processes and pathways altered in response to different EPF treatments. Changes in proteins involved in molecular and biological processes, as annotated by Blast2GO, is shown in Fig. 4.

\section{Discussion}

The aims of the experiments described here were to investigate immunomodulatory effects of EPF culture filtrate treatments on a model insect and specifically to determine whether EPF treatment rendered the insect more susceptible to subsequent pathogen attack. Understanding how insects resist and/or detoxify EPF secretory products and identifying the specific biological responses elicited in susceptible hosts is essential to the development of effective fungal biological control strategies (Rohlfs and Churchill, 2011). Our results give insight into the effect EPF culture filtrate has on the cellular and humoral immune response of $G$. mellonella larvae and provide information regarding the specific immunomodulation potential of EPF. $M$. anisopliae and B. caledonica $96 \mathrm{~h}$ culture filtrates exhibited clear immunomodulatory effects on $G$. mellonella larvae, increasing their susceptibility to pathogenic infection, whereas $B$. bassiana filtrate had no significant effect on susceptibility to pathogens.

4.1. B. caledonica, B. bassiana and M. anisopliae culture filtrates induce varied immune responses in $G$. mellonella

Culture filtrate of all three EPF caused a significant decline in the haemocyte density of larvae. Haemocyte death is important in innate immunity as haemocytes are associated with encapsulation and nodulation (Butt et al., 2016). Treatment with B. caledonica and M. anisopliae $96 \mathrm{~h}$ culture filtrate facilitated a significantly increased yeast cell density in larvae suggesting that filtrate components are modulating the immune system of the insect allowing a subsequent pathogen to proliferate. In contrast, treatment with $B$. bassiana culture filtrate did not allow a significant increase in yeast cell density in larvae relative to controls. Larvae co-injected with $M$. anisopliae or B. caledonica culture filtrate and $C$. albicans showed significantly increased mortality. The proposed immunomodulation that is rendering the host more susceptible to a subsequent pathogen is presumably caused by spore free culture filtrate, known to contain a diverse mixture of enzymes, proteases and secondary metabolites. Destruxin, the most abundantly 

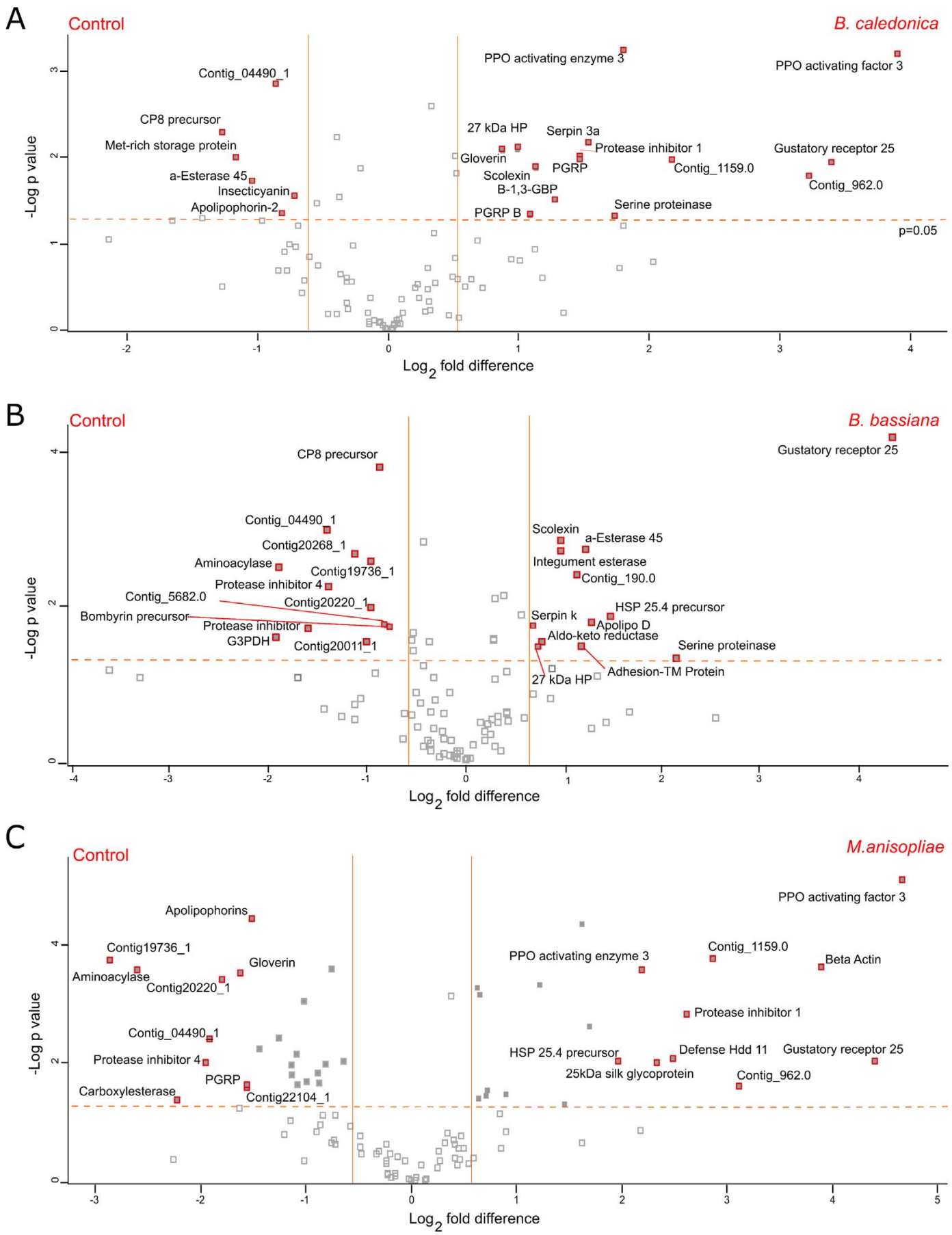

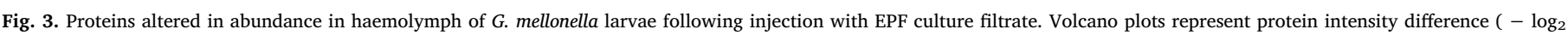

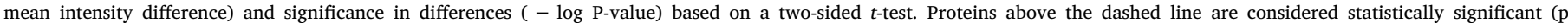

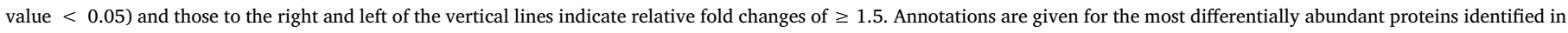

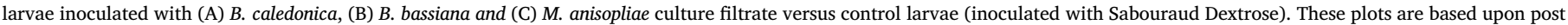
imputed data.

produced secondary metabolite in Metarhizium spp., induced a similar response in D. melanogaster (Pal et al., 2007).

Proteomic analysis indicates that the response to the culture filtrate from $M$. anisopliae was the most divergent relative to the control, although some similarities in proteome alteration were observed between $B$. caledonica and M. anisopliae. This observation complements the bioassay results that show effects of $B$. caledonica are more similar to $M$. anisopliae than to $B$. bassiana. GO term annotation identified processes and pathways altered in response to the different EPF treatments with the $M$. anisopliae culture filtrate appearing to induce changes in the largest number of processes, including protein, lipid and ion binding.

$B$. bassiana treated larvae displayed higher abundances of alphaesterase when compared to control larvae and larvae treated with $M$. anisopliae and B. caledonica culture filtrate. Insects infected with EPF often upregulate antioxidant genes, and fungal infection of insects is associated with increased total esterase and glutathione S-transferase activities in the hemolymph (Butt et al., 2016). Increased activity of detoxification enzymes represents the insect immune response to intoxication with metabolites produced by the pathogen or with 
A
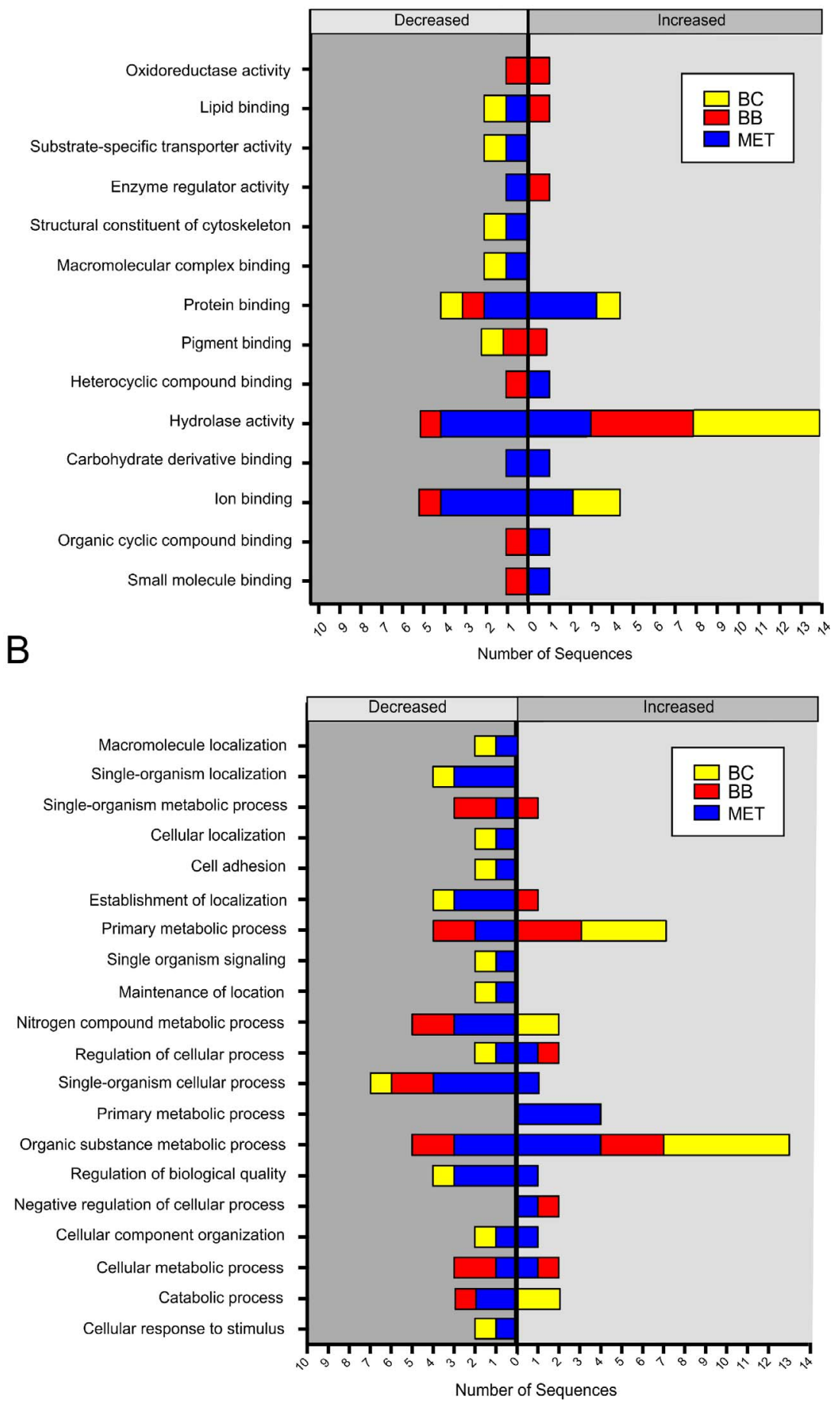

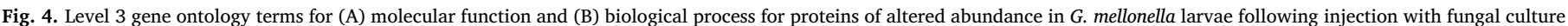
filtrate.

products of host tissue degradation. Conversely, inhibition of detoxification enzymes increases insect death rate from fungal infection (Serebrov et al., 2006). Induction of additional esterase isoforms and increased glutathione S-transferase activity in G. mellonella larvae with mycoses can decrease their sensitivity to chemical insecticides (Hemingway and Ranson, 2000; Serebrov et al., 2006). A lethal dose of $M$. anisopliae was found to increase activity of detoxifying enzymes in the locust nymphs on the third day after infection, and a reduction in esterase and GST activity during acute mycosis was linked with effective inhibition of the host defense systems (Dubovskiy et al.,
2012). Alpha esterase is decreased in abundance in B. caledonica treated larvae relative to control larvae, while alpha esterase and carboxylesterase is decreased in expression in $M$. anisopliae treated larvae. Thus it could be postulated that detoxifying enzymes induced by treatment with $B$. bassiana culture filtrate may have had some role in the reduction of susceptibility of those larvae to subsequent pathogen attack. 


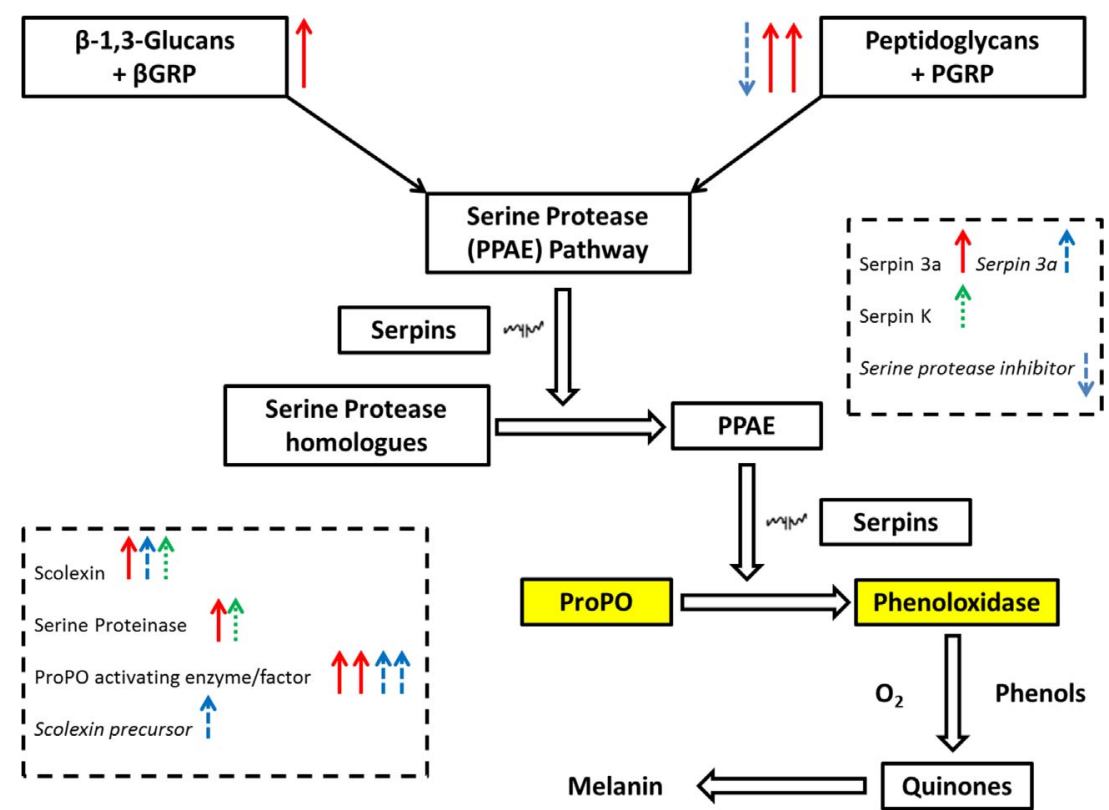

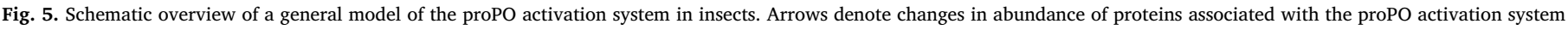

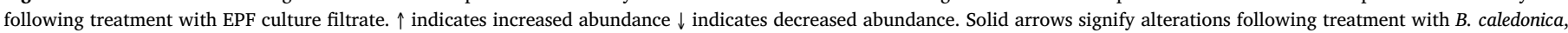

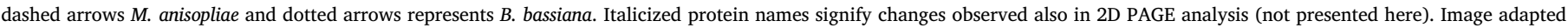
from González-Santoyo and Córdoba-Aguilar (2012).

\subsection{B. caledonica and M. anisopliae culture filtrates impact upon the ProPO cascade in G. mellonella}

LFQ analysis of the effect of $M$. anisopliae and $B$. caledonica culture filtrate on the proteome of $G$. mellonella hemolymph demonstrated an alteration in abundance of proteins implicated in the proPO (prophenoloxidase) cascade (summarised in Fig. 5), in both regulation and maintenance of PO (phenoloxidase). ProPO activating factor 3 (PPAF 3 ), proPO activating enzyme 3 (PPAE 3 ), serpin $3 \mathrm{a}$, serine proteinase, protease inhibitor 1 precursor and scolexin were all increased in expression in larvae injected with $B$. caledonica filtrate. PO activities are vital for early host responses to EPF on the cuticle, during encapsulation responses to EPF in the haemocoel and for production of melanin and reactive oxygen and nitrogen species. Serine proteinases and the molecules they produce can be damaging to both the insect host and the fungal pathogen, affecting nucleic acids, peptides and oxidation of lipids (Jiang et al., 2010; Butt et al., 2016). Serpin 3 is increased in abundance following treatment with $B$. caledonica. Many serpins are irreversible inhibitors, key modulators of the proPO cascade and deactivators of microbe-derived proteases (Jiang et al., 2010; Butt et al., 2016). Serpin 3 was found to be constitutively expressed in $M$. sexta at low levels and increased in expression after microbial challenge. Recombinant serpin 3 blocks proPO activation in hemolymph and forms complexes with proPO activating proteinases (Zhu et al., 2003). Together these alterations in proPO pathway proteins (Fig. 5), indicate both a response in the insect to the treatment and an attempt to prevent the deleterious effects of uncontrolled PO activity (Sadd and Siva-Jothy, 2006).

A number of similarities were observed in the proteome response to $B$. caledonica and $M$. anisopliae culture filtrates which may explain similar susceptibilities to subsequent pathogens. ProPO activating factor 3 , protease inhibitor 1 and proPO activating enzyme 3 , for example, had increased abundance in B. caledonica and M. anisopliae treated larvae. Protease inhibitor 4 was downregulated in larvae treated with $M$. anisopliae and B. bassiana. Scolexin was observed in higher abundance in larvae following administration of all three EPF filtrates, but at a higher fold change in M. anisopliae and B. caledonica. Thus it seems that there is a clear similarity in proPO cascade protein profiles in larvae treated with the EPF filtrates, which may explain the similarity in pathogen susceptibility. This immunomodulation potential of EPF on the insect immune response may have implications for the utilisation of EPF as a biocontrol agent. The ability of EPF to suppress the insect immune system making it more susceptible to subsequent pathogens may potentially enhance EPF ability to act synergistically with other control agents in the field.

\subsection{EPF culture filtrate alters abundance of pathogen recognition proteins in G. mellonella}

Administration of three EPF culture filtrates resulted in increased abundance in a gustatory protein in the insect haemolymph. This may be linked to the fact that the production and maintenance of the proPO activating pathway is diet-dependent (Siva-Jothy and Thompson, 2002; González-Santoyo and Córdoba-Aguilar, 2012) and as there are changes to proteins related to the proPO pathway induced by all three EPF it may be necessary for the larvae to increase their dietary intake to sustain the cascade. The cost of activating and using immune defenses can itself reduce host fitness. Hosts may compensate for increased demands by increased resource uptake (Moret and Schmid-Hempel, 2000). A different explanation may be found in the work of Lee et al. (2010). They demonstrated that Drosophila can detect minute $(0.05 \%)$ concentrations of DEET, the most widely used insect repellent worldwide, through the gustatory response and that it suppressed feeding. This behavior was mediated by direct activation of avoidance gustatory receptor neurons. Compounds that inhibit feeding have been shown to stimulate gustatory receptor cells in the mouthpart sensilla of certain orthopteran and coleopteran insects and a number of larval Lepidoptera (Chapman, 2003), highlighting the importance of gustatory receptors in toxin avoidance.

Administration of EPF culture filtrate to G. mellonella larvae impacted upon proteins instrumental in pathogen recognition that ultimately lead to activation of an appropriate immune response. Insects differentiate between major groups of microbes using PRRs such as peptidoglycan recognition proteins (PGRPs), hemolin and $\beta-1,3-$ glucan binding protein ( $\beta$ GBPs). PRRs function by binding to PAMPs on microbial cells such as $\beta-1,3$-glucan from fungi that acts as a signal to activate the antifungal functions of Toll (Stokes et al., 2015). Three proteins involved in recognition were increased in abundance following 
treatment with $B$. caledonica: $\beta$ GBP and two PGRPs. These receptors are crucial to recognition of pathogens and activation of an appropriate immune response (e.g. proPO pathway). Both $M$. anisopliae and $B$. caledonica treated larvae display a lower abundance of apolipophorins which in addition to lipid transport can function as pattern recognition receptors by binding to bacterial and fungal cell walls (ZdybickaBarabas and Cytryńska, 2013). M. anisopliae and B. caledonica treated larvae have a lower abundance of apolipophorins than larvae treated with $B$. bassiana or controls. In addition a PRR peptidoglycan recognition-like protein was observed in all samples except $M$. anisopliae treated larvae where it was undetected (essentially absent). These receptors are crucial to recognition of pathogens and the activation of an appropriate immune response such as the proPO pathway.

\subsection{EPF culture filtrate alters antimicrobial peptide production in $G$. mellonella}

Treatment with EPF culture filtrate had a significant effect on AMP abundances particularly in $M$. anisopliae treated larvae where cecropin$\mathrm{D}$ precursor, hemolin and gloverin were differentially abundant in comparison to controls (Table 1 and 2). Cecropin-D precursor, a member of the $4 \mathrm{kDa}$ cecropins found in Lepidoptera and Diptera, was detected in the haemolymph of all treatments except in those larvae treated with $M$. anisopliae culture filtrate. These proteins are membrane active antibiotics that act on Gram-positive and -negative bacteria by forming channels that permeabilise the lipid bilayer (Hoffmann, 1995; Jiang et al., 2010). Some cecropins also demonstrate antifungal activity (Faruck et al., 2015). Hemolin was only significantly increased in expression in $M$. anisopliae treated larvae. Hemolin is a $47 \mathrm{kDa}$ hemolymph protein present at a low constitutive level that is increased upon bacterial challenge. It is thought to have a role in immune recognition and in modulation of defensive responses in $H$. cecropia and M. sexta. Hemolin has a role in antibacterial defense indicated by its structural similarity to cell adhesion molecules and its increased expression induced by bacteria. Hemolin can bind to surfaces of bacteria and haemocytes and can stimulate the phagocytic activity of haemocytes (Gillespie et al., 1997, 2000). A glycine rich AMP peptide gloverin was significantly decreased in expression in $M$. anisopliae treated larvae only. Glycine rich peptides have antifungal activity and are active against yeasts (Faruck et al., 2015) so their downregulation may contribute in part to the increased proliferation of $C$. albicans in larvae pretreated with $M$. anisopliae culture filtrate. Gloverin was increased in abundance in larvae treated with $B$. caledonica culture filtrate. This AMP is effective against Gram-negative bacteria. Gloverin acts by inhibiting the synthesis of vital outer membrane proteins resulting in an increase in permeability of the outer membrane of the bacteria (Bulet et al., 1999). One implication of an upregulation in AMP active against bacteria, is that antibacterial activities can be highly beneficial to EPF as it might help exclude opportunistic infections that would be disadvantageous to the fungus (Butt et al., 2016).

The lower abundance of proteins, such as cecropin and PRR, that are instrumental in the insect's ability to recognise and respond to pathogens are indicative of $M$. anisopliae immunomodulation potential observed in bioassays. The immune responses in this work are induced by cell- and spore-free culture filtrate which contains many secreted products. A direct relationship has previously been established between destruxin production and the virulence of $M$. anisopliae. Pal et al. (2007) injected Drosophila with destruxin that lead to a reduction in expression of AMP genes including Cecropin. Co-injection of destruxin with $E$. coli caused a significant decrease in survival of Drosophila. Thus it was suggested that a lowering in AMP production induced by destruxin allows $E$. coli to proliferate and colonize the fly to accelerate its demise (Pal et al., 2007). Through selectively reducing bacterial AMPs with the secretion of destruxin $M$. anisopliae may create an advantageous environment where bacteria can proliferate and, thereby, contribute to accelerating the demise of its insect host (Rohlfs and Churchill,
2011). Pal et al. (2007) complements the observation in this work that treatment with $M$. anisopliae culture filtrate causes a reduction in cecropin abundance among other immune relevant proteins and in parallel there is increased yeast proliferation and mortality when $G$. mellonella are injected with fungal culture filtrate before injection with C. albicans. Thus suggesting that EPF culture filtrate and the secreted products contained within can suppress the immune response of insects.

\section{Conclusion}

Understanding how EPF modulate the immune response leaving insects more susceptible to subsequent pathogens may have application in selecting superior strains with these characteristics to overcome problems with EPF killing target pests too slowly or inefficiently compared to their chemical counterparts. Additionally, EPF isolates could be screened for their ability to produce particular secreted products that induce immunomodulation in target insects. This aim of this work was to investigate the effect of culture filtrates from three EPF species (two widely used EPF and one less well studied species) on the insect immune response using $G$. mellonella larvae as a model system. The immune responses induced in G. mellonella were in response to injection with spore free culture filtrate, so it is a reflection of the immune response induced by EPF secreted products. A number of fungal secreted products are known to be important virulence determinants. It has been previously demonstrated that abundant EPF secreted products such as destruxin and oosporein induce changes to immune response of insects affecting AMP and the proPO cascade as well as the cellular immune response. These findings aid in understanding how the desired synergism between biocontrol agents could mechanistically occur e.g. interfering with the proPO cascade and the production of AMP.

Bioassays allowed assessment of the immunomodulation of different treatments and proteomic analysis aided in understanding mechanistically how these variations may have occurred e.g. alterations to proteins/pathways that may render the insect more susceptible to subsequent pathogens. The results indicate that $M$. anisopliae and $B$. caledonica culture filtrate exhibit an immunomodulation effect on $G$. mellonella larvae. Together the bioassay results and proteomic profiling indicate that $M$. anisopliae culture filtrate has the greatest impact on the insect immune system. Differences in proteomic profiles for different treatments may reflect or even partial explain the differences observed in bioassays. For example, higher levels of detoxifying enzymes in $B$. bassiana treated larvae than in those treated with $M$. anisopliae and $B$. caledonica may be one alteration reflective of their differences in immunomodulation. This proteomic work gives an insight into the mechanisms behind the observed differences in immunomodulation of the tested EPF and is intended to fit into the wider investigation of EPF immunomodulation in insects.

\section{Disclosure statement}

No potential conflict of interest was reported by the authors.

\section{Funding}

L. Mc Namara was funded by the Irish Government (Department of Agriculture, Food and the Marine) under the National Development Plan 2007-2013. The Q-Exactive quantitative mass spectrometer was funded under the SFI Research Infrastructure Call 2012; Grant No.: 12/ $\mathrm{RI} / 2346$ (3).

\section{Acknowledgments}

We thank Caroline Batchelor for her assistance with the Q-Exactive quantitative mass spectrometer. 


\section{Appendix A. Supplementary data}

All proteins identified from the haemolymph of EPF supernatant treated and control Galleria mellonella larvae. Statistically significantly differentially abundant proteins ( 2 sample $t$-tests; $\mathrm{p}<0.05$ ) and relative fold change differences for all comparisons for the post imputed data set and the exclusively expressed proteins (pre imputation) are also given.

Supplementary data associated with this article can be found, in the online version, at http://dx.doi.org/10.1016/j.jinsphys.2017.05.009.

\section{References}

Amiri-Besheli, B., Khambay, B., Cameron, S., Deadman, M.L., Butt, T.M., 2000. Inter-and intra-specific variation in destruxin production by insect pathogenic Metarhizium spp., and its significance to pathogenesis. Mycol. Res. 104 (04), 447-452.

Anbesse, S.A., Adge, B.J., Gebru, W.M., 2008. Laboratory screening for virulent entomopathogenic nematodes (Heterorhabditis bacteriophora and Steinernema yirgalemense) and fungi (Metarhizium anisopliae and Beauveria bassiana) and assessment of possible synergistic effects of combined use against grubs of the barley chafer Coptognathus curtipennis. Nematology 10 (5), 701-709.

Ansari, M.A., Shah, F.A., Tirry, L., Moens, M., 2006. Field trials against Hoplia philanthus (Coleoptera: Scarabaeidae) with a combination of an entomopathogenic nematode and the fungus Metarhizium anisopliae CLO 53. Biol. Control 39 (3), 453-459.

Ansari, M.A., Shah, F.A., Butt, T.M., 2008. Combined use of entomopathogenic nematodes and Metarhizium anisopliae as a new approach for black vine weevil, Otiorhynchus sulcatus, control. Entomol. Exp. Appl. 129 (3), 340-347.

Ansari, M.A., Shah, F.A., Butt, T.M., 2010. The entomopathogenic nematode Steinernema kraussei and Metarhizium anisopliae work synergistically in controlling overwintering larvae of the black vine weevil, Otiorhynchus sulcatus, in strawberry growbags. Biocontrol Sci. Tech. 20 (1), 99-105.

Bergin, D., Brennan, M., Kavanagh, K., 2003. Fluctuations in haemocyte density and microbial load may be used as indicators of fungal pathogenicity in larvae of Galleria mellonella. Microbes Infect. 5 (15), 1389-1395.

Bergin, D., Murphy, L., Keenan, J., Clynes, M., Kavanagh, K., 2006. Pre-exposure to yeast protects larvae of Galleria mellonella from a subsequent lethal infection by Candida albicans and is mediated by the increased expression of antimicrobial peptides. Microbes Infect. 8 (8), 2105-2112.

Browne, N., Surlis, C., Maher, A., Gallagher, C., Carolan, J.C., Clynes, M., Kavanagh, K., 2015. Prolonged pre-incubation increases the susceptibility of Galleria mellonella larvae to bacterial and fungal infection. Virulence 6 (5), 458-465.

Bulet, P., Hetru, C., Dimarcq, J.-L., Hoffmann, D., 1999. Antimicrobial peptides in insects; structure and function. Dev. Comp. Immunol. 23 (4), 329-344.

Butt, T.M., Coates, C.J., Dubovskiy, I.M., Ratcliffe, N.A., 2016. Entomopathogenic fungi: new insights into host-pathogen interactions. Adv. Genet. 94, 307-364.

Chapman, R.F., 2003. Contact chemoreception in feeding by phytophagous insects. Annu. Rev. Entomol. 48 (1), 455-484.

Côté, R.G., Griss, J., Dianes, J.A., Wang, R., Wright, J.C., van den Toorn, H.W., van Breukelen, B., Heck, A.J., Hulstaert, N., Martens, L., Reisinger, F., Csordas, A., Ovelleiro, D., Perez-Rivevol, Y., Barsnes, H., Hermjakob, H., Vizcaíno, J.A., 2012. The PRoteomics IDEntification (PRIDE) Converter 2 framework: an improved suite of tools to facilitate data submission to the PRIDE database and the ProteomeXchange consortium. Mol. Cell. Proteomics 11 (12), 1682-1689.

Cotter, G., Doyle, S., Kavanagh, K., 2000. Development of an insect model for the in vivo pathogenicity testing of yeasts. FEMS Immunol. Med. Microbiol. 27 (2), 163-169.

Dubovskiy, I.M., Slyamova, N.D., Kryukov, V.Y., Yaroslavtseva, O.N., Levchenko, M.V., Belgibaeva, A.B., Adilkhankyzy, A., Glupov, V.V., 2012. The activity of nonspecific esterases and glutathione-S-transferase in Locusta migratoria larvae infected with the fungus Metarhizium anisopliae (Ascomycota, Hypocreales). Entomol. Rev. 92 (1), $27-31$.

Faruck, M.O., Yusof, F., Chowdhury, S., 2015. An overview of antifungal peptides derived from insect. Peptides 80, 80-88.

Gao, Q., Jin, K., Ying, S.-H., Zhang, Y., Xiao, G., Shang, Y., Duan, Z., Hu, X., Xie, X.-Q., Zhou, G., 2011. Genome sequencing and comparative transcriptomics of the model entomopathogenic fungi Metarhizium anisopliae and M. acridum. PLoS Genet. 7 (1), e1001264.

Gillespie, J.P., Kanost, M.R., Trenczek, T., 1997. Biological mediators of insect immunity. Annu. Rev. Entomol. 42 (1), 611-643.

Gillespie, J.P., Bailey, A.M., Cobb, B., Vilcinskas, A., 2000. Fungi as elicitors of insect immune responses. Arch. Insect Biochem. Physiol. 44 (2), $49-68$.

Glare, T.R., Reay, S.D., Nelson, T.L., Moore, R., 2008. Beauveria caledonica is a naturally occurring pathogen of forest beetles. Mycol. Res. 112 (3), 352-360.

González-Santoyo, I., Córdoba-Aguilar, A., 2012. Phenoloxidase: a key component of the insect immune system. Entomol. Exp. Appl. 142 (1), 1-16.

Hemingway, J., Ranson, H., 2000. Insecticide resistance in insect vectors of human disease. Annu. Rev. Entomol. 45 (1), 371-391.

Hoffmann, J.A., 1995. Innate immunity of insects. Curr. Opin. Immunol. 7 (1), 4-10.

Hung, S.-Y., Boucias, D.G., Vey, A.J., 1993. Effect of Beauveria bassiana and Candida albicans on the cellular defense response of Spodoptera exigua. J. Invertebr. Pathol. 61 (2), 179-187.

Inglis, G.D., Johnson, D.L., Cheng, K.J., Goettel, M.S., 1997. Use of pathogen combinations to overcome the constraints of temperature on entomopathogenic hyphomycetes against grasshoppers. Biol. Control 8 (2), 143-152.

Jiang, H., Vilcinskas, A., Kanost, M.R., 2010. Immunity in lepidopteran insects. Invertebrate Immunity. Springerpp. 181-204.

Kershaw, M.J., Moorhouse, E.R., Bateman, R., Reynolds, S.E., Charnley, A.K., 1999. The role of destruxins in the pathogenicity of Metarhizium anisopliae for three species of insect. J. Invertebr. Pathol. 74 (3), 213-223.

Lacey, L.A., Grzywacz, D., Shapiro-Ilan, D.I., Frutos, R., Brownbridge, M., Goettel, M.S. 2015. Insect pathogens as biological control agents: back to the future. J. Invertebr. Pathol. 132, 1-41.

Lee, Y., Kim, S.H., Montell, C., 2010. Avoiding DEET through insect gustatory receptors. Neuron 67 (4), 555-561.

Leger, R.J.S., Wang, C., Fang, W., 2011. New perspectives on insect pathogens. Fungal Biol. Rev. 25 (2), 84-88.

Liu, B.L., Tzeng, Y.M., 2012. Development and applications of destruxins: a review. Biotechnol. Adv. 30 (6), 1242-1254.

Moret, Y., Schmid-Hempel, P., 2000. Survival for immunity: the price of immune system activation for bumblebee workers. Science 290 (5494), 1166-1168.

Pal, S., Leger, R.J.S., Wu, L.P., 2007. Fungal peptide Destruxin A plays a specific role in suppressing the innate immune response in Drosophila melanogaster. J. Biol. Chem. 282 (12), 8969-8977.

Reay, S.D., Brownbridge, M., Cummings, N.J., Nelson, T.L., Souffre, B., Lignon, C., Glare, T.R., 2008. Isolation and characterization of Beauveria spp. associated with exotic bark beetles in New Zealand Pinusradiata plantation forests. Biol. Control 46 (3), 484-494.

Roberts, D.W., St Leger, R.J., 2004. Metarhizium spp., cosmopolitan insect-pathogenic fungi: mycological aspects. Adv. Appl. Microbiol. 54, 1-70.

Rohlfs, M., Churchill, A.C.L., 2011. Fungal secondary metabolites as modulators of interactions with insects and other arthropods. Fungal Genet. Biol. 48 (1), 23-34.

Sadd, B.M., Siva-Jothy, M.T., 2006. Self-harm caused by an insect's innate immunity. Proc. R. Soc. London B Biol. Sci. 273 (1600), 2571-2574.

Schrank, A., Vainstein, M.H., 2010. Metarhizium anisopliae enzymes and toxins. Toxicon 56 (7), 1267-1274.

Scully, L.R., Bidochka, M.J., 2005. Serial passage of the opportunistic pathogen Aspergillus flavus through an insect host yields decreased saprobic capacity. Can. J. Microbiol. 51 (2), 185-189.

Serebrov, V.V., Gerber, O.N., Malyarchuk, A.A., Martemyanov, V.V., Alekseev, A.A., Glupov, V.V., 2006. Effect of entomopathogenic fungi on detoxification enzyme activity in greater wax moth Galleria mellonella L. (Lepidoptera, Pyralidae) and role of detoxification enzymes in development of insect resistance to entomopathogenic fungi. Biology Bulletin 33 (6), 581-586.

Shah, P.A., Pell, J.K., 2003. Entomopathogenic fungi as biological control agents. Appl. Microbiol. Biotechnol. 61 (5-6), 413-423.

Siva-Jothy, M.T., Thompson, J.J.W., 2002. Short-term nutrient deprivation affects immune function. Physiol. Entomol. 27 (3), 206-212.

Sowjanya Sree, K., Padmaja, V., Murthy, Y.L.N., 2008. Insecticidal activity of destruxin, a mycotoxin from Metarhizium anisopliae (Hypocreales), against Spodoptera litura (Lepidoptera: Noctuidae) larval stages. Pest Manag. Sci. 64 (2), 119-125.

Stokes, B.A., Yadav, S., Shokal, U., Smith, L.C., Eleftherianos, I., 2015. Bacterial and fungal pattern recognition receptors in homologous innate signaling pathways of insects and mammals. Front. Microbiol. 6, 19.

van der Weerden, N.L., Bleackley, M.R., Anderson, M.A., 2013. Properties and mechanisms of action of naturally occurring antifungal peptides. Cell. Mol. Life Sci. 70 (19), 3545-3570.

Vilcinskas, A., Götz, P., 1999. Parasitic fungi and their interactions with the insect immune system. Adv. Parasitol. 43, 267-313.

Vilcinskas, A., Matha, V., 1997. Effect of the entomopathogenic fungus Beauveria bassiana on the humoral immune response of Galleria mellonella larvae (Lepidoptera: Pyralidae). Eur. J. Entomol. 94, 461-472.

Vilcinskas, A., Matha, V., Götz, P., 1997a. Effects of the entomopathogenic fungus Metarhizium anisopliae and its secondary metabolites on morphology and cytoskeleton of plasmatocytes isolated from the greater wax moth, Galleria mellonella. J. Insect Physiol. 43 (12), 1149-1159.

Vilcinskas, A., Matha, V., Götz, P., 1997b. Inhibition of phagocytic activity of plasmatocytes isolated from Galleria mellonella by entomogenous fungi and their secondary metabolites. J. Insect Physiol. 43 (5), 475-483.

Vogel, H., Altincicek, B., Glöckner, G., Vilcinskas, A., 2011. A comprehensive transcriptome and immune-gene repertoire of the lepidopteran model host Galleria mellonella. BMC Genomics 12 (1), 308.

Williams, C.D., Dillon, A.B., Harvey, C.D., Hennessy, R., Mc Namara, L., Griffin, C.T., 2013. Control of a major pest of forestry, Hylobius abietis, with entomopathogenic nematodes and fungi using eradicant and prophylactic strategies. Forest Ecol. Manage. 305, 212-222.

Wojda, I., Jakubowicz, T., 2007. Humoral immune response upon mild heat-shock conditions in Galleria mellonella larvae. J. Insect Physiol. 53 (11), 1134-1144.

Wojda, I., Kowalski, P., Jakubowicz, T., 2009. Humoral immune response of Galleria mellonella larvae after infection by Beauveria bassiana under optimal and heat-shock conditions. J. Insect Physiol. 55 (6), 525-531.

Xiao, G., Ying, S.-H., Zheng, P., Wang, Z.-L., Zhang, S., Xie, X.-Q., Shang, Y., Leger, R.J.S., Zhao, G.-P., Wang, C., 2012. Genomic perspectives on the evolution of fungal entomopathogenicity in Beauveria bassiana. Sci. Rep. 2, 483.

Zdybicka-Barabas, A., Cytryńska, M., 2013. Apolipophorins and insect immune response. Invertebrate Surv. J. 10, 58-68.

Zhu, Y., Wang, Y., Gorman, M.J., Jiang, H., Kanost, M.R., 2003. Manduca sexta serpin-3 regulates prophenoloxidase activation in response to infection by inhibiting prophenoloxidase-activating proteinases. J. Biol. Chem. 278 (47), 46556-46564. 